magnetic field is an asymmetric tensor. There is no system of three principal directions for which the current density components would be proportional to the electric field strength components. This simple proportionality is however found in a coordinate system whose coordinates are a vector in the direction of the magnetic field and two vectors rotating in either sense around the magnetic field direction. The longitudinal conductivity, that is, the conductivity in the magnetic field direction, is predominant.
The transverse conductivities now will be assumed being suppressed by the magnetic field. The propagation characteristics then become independent of the electron concentration. The two magnetoionic modes in this case have refractive indices of or near 1 , their attenuation is negligible, and one of them is perfectly guided along the magnetic field lines. This peculiar type of propagation requires a magnetic field of sufficient intensity, but no influence of ion motions or pressure gradients in the plasma.

\title{
NON-LINEAR MODELLING OF MAXWELL'S EQUATIONS
}

\section{J. E. BELYEA and $K$. M. SIEGEL University of Michigan}

The basic stumbling block to carrying out many experiments in the laboratory is the reliance which is placed in the theory of linear modelling. In aerodynamics and electromagnetics one is seldom able to construct a precise linear model; one must appeal to physical reasoning to show that the model one is able to construct gives results in close agreement with those which would have been obtained from a precise one. For example, as long as good conductors are used, conductivity is not modelled.

Up until recent years the limitation on indoor radar cross-section ranges was simply the size of the model that could be handled and the magnitude of the frequency of the coherent source. No precise linear modelling experiments exist for bomber cross-sections at very high microwave frequencies.

With the growth of interest in ICBM pro- grammes it becomes desirous (as measured in dollars) to determine the radar cross-sections of ablating warheads, and to determine attentuation effects and scattering effects in or by plasmas, by modelling. Linear modelling is of very limited utility for such experiments. One must model a lossy dielectric at high frequenc1es or not do the experiment in the laboratory. One must model some plasma parameters, or risk missing crucial first order effects.

Previous analysis predicted the feasibility of non-linear modelling. The first such modelling results are given in Ref. (1). In this paper many non-linear models of equations of mathematical physics are presented. Maxwell's equations are non-linearly modelled. Experiments making use of non-linear models are discussed.

1. R. K. Rurt, Trans. Inst. Radio Engrs. on Antennas and Propagation, 4, 216 (1956).

\section{THE REGION BEHIND A BODY MOVING THROUGH A RAREFIED IONIZED ATMOSPHERE}

\author{
E. T. KOROWSKI \\ General Electric Company
}

Physical arguments indicate that charge density gradients will exist some distance behind and possibly a short distance to the side of a body moving at high speed through the ionized layers of the upper atmosphere.

The medium is taken to be highly rarefied gas 